\title{
Mining-Associated Seismicity in Kolar Gold Mines- Some Case Studies Using Multifractals
}

\author{
S. Karekal CRC Mining and the School of Engineering (Mining Engineering Program), University of Queensland, Australia \\ M.V.M.S. Rao National Geophysical Research Institute, India \\ S. Chinnappa National Institute of Rock Mechanics, Kolar Gold Fields, India
}

The occurrence of rockbursts was quite common during active mining periods in the Champion reef mines of Kolar gold fields, India. Among the major rockbursts, the 'area-rockbursts' were unique both in regard to their spatio-temporal distribution and the extent of damage caused to the mine workings. A detailed study of the spatial clustering of 3 major area-rockbursts $(A R B)$ was carried out using a multi-fractal technique involving generalized correlation integral functions. The spatial distribution analysis of all 3 area-rockbursts showed that they are heterogeneous. The degree of heterogeneity $\left(D_{2}-D_{\alpha}\right)$ in the cases of ARB-I, II and III were found to be 0.52, 0.37 and 0.41 respectively. These differences in fractal structure indicate that the ARBs of the present study were fully controlled by different heterogeneous stress fields associated with different mining and geological conditions. The present study clearly showed the advantages of the application of multi-fractals to seismic data and to characterise, analyse and examine the area-rockbursts and their causative factors in the Kolar gold mines.

\section{INTRODUCTION}

Rockbursts are caused essentially by the alteration of the virgin state of stress or rockmass equilibrium due to mining processes (Cook, 1976). The large strain energy accumulation in the rockmass, and sudden release of this energy in the form of violent, and dynamic manner are called rockbursts.

The occurrence of rockbursts (or mine tremors) has been a common feature in the century-old mines of Kolar Gold Fields (KGF), which are situated in the schist belt of the southern Indian peninsular shield. These gold mines were once the main source of gold in the country, which enjoyed the distinction of being amongst the few oldest and deepest mines of the world. Some of the workings reached a maximum depth of about $3.3 \mathrm{~km}$ below the ground level. Due to economic constraints in the extraction of ore, the deeper levels were discontinued towards the end of 1991, and the shallow workings have been shutdown recently in the Champion reef mine. The relatively rich ore body and working stopes at great depths $(\sim 9,000$ to $10,000 \mathrm{ft}$ or 2,727 to $3,030 \mathrm{~m}$ ) in that mine were found associated with large size faults and dykes resulting in high stress concentrations and the occurrence of major rockbursts. Among the major rockbursts, the 'area-rockbursts' were unique both in regard to their spatio-temporal distribution and the extent of damage caused to the mine workings (Miller 1967, Krishnamurthy 1977, 1986, Krishnamurthy and Shringarputale 1990. These 'area-rockbursts' were produced when a major rockburst of local magnitude 3.0 (on the Richter Scale) triggered a cluster series of 20 - 200 bursts of smaller magnitude in close succession in time in active mine workings and / or old (or suspended) mine workings. The hypocenters of an arearockburst are generally concentrated over a larger region. These area-rockbursts have caused fatalities and extensive damage to the surface and underground mine structures. Many attempts have been made in the past to predict the occurrence of these area-rockbursts, but the success achieved has been limited due to the complex nature of the rockmass and the complex physical process of rockbursts.

The analysis of natural and induced seismicity has received a new impetus in recent years with the application of 'Fractals'. The concept of fractal geometry was originally introduced by Mandelbrot (1982) to describe the irregular and non-smooth phenomena in nature. Since then many remarkable advances have been made in describing and interpreting complex phenomena in geo-sciences. The basis of fractal structure is the self-similarity or the scale-invariant characteristics. Studies have shown that the fracture process of rock, including earthquakes, has a typical fractal structure in space, time and magnitude distribution as expressed by the fractal dimension, $\mathrm{D}$, Omari's exponent, $\mathrm{P}$ and the $\mathrm{b}$ value, respectively (Hirata et al. 1987, Mogi 1962, Scholz 1968). Attempts have been made to apply the fractal techniques to understand and predict rockburst by studying spatial and temporal distribution of microseismic events (Coughlin and Kranz 1991, Xie and Pariseau 1992). In their analysis, they considered the distribution of microseismic events as a homogeneous fractal. In reality, many natural phenomena do not have perfect homogeneous scale-invariant characteristics described by a single fractal dimension. In most cases, they are bounded and heterogeneous in space (Hirata and Imoto 1991, Lei et al. 1993). In such cases the multifractal concept, which is a natural extension of the fractal concept to heterogenous fractals, would be more useful for analysis of the fine structure of the spatial pattern of rockbursts.

This paper describes the stochastic self-similar concept of the spatial distribution of hypocenters of three major arearockbursts from the multifractal perspective to characterise, analyse and examine the area-rockbursts and their causative factors.

\section{GEOLOGY AND IN SITU STRESSES}

The Gold mines of KGF are located in highly metamorphosed hornblende schists, having a general north-south strike and dipping west. The Kolar Schist belt is $80 \mathrm{~km}$ long in the northsouth direction and about $4 \mathrm{~km}$ wide in the east-west direction, with the major mining activities in the southern side (Fig. 1). The schists are folded, faulted and intruded by dolerite and porphyry dykes and pegmatite intrusions. The schists are generally homogeneous, highly crystalline and brittle. There are a number of gold bearing quartz reefs, which dip near the surface at $40^{\circ}-50^{\circ}$ towards west and gradually become steeper, until they are nearly vertical at depth. Only two of the reefs, 
namely the Champion and the Oriental, were of economic grade. In both the dip and the strike directions, the reefs were practically parallel to the foliation plane of the schists. The areal extent of Kolar Gold mines is about $8.5 \mathrm{~km}$ in the North-South (N-S) direction along the main longitudinal axis of the mining region and about $3.5 \mathrm{~km}$ across it. The mining region is traversed by three geological fault systems with the prominent one striking NW-SE right through the centre of the region and is known as Mysore North Fault (MNF). The other two, namely Tennant Fault and Gifford Fault, are minor faults subparallel to MNF.

In situ stresses, measured at depths of $590 \mathrm{~m}$ and $1044 \mathrm{~m}$ using hydraulic fracturing technique, are given in Table 1 (Gowd et al. 1981). The major horizontal principal stress was established to be oriented NW-SE at both the levels.

TABLE 1 In situ principal stresses at Champion reef mine

\begin{tabular}{llll}
\hline Depth $(\mathrm{m})$ & $\begin{array}{l}\text { Major } \\
\text { horizontal } \\
\text { principal } \\
\text { stress (MPa) }\end{array}$ & $\begin{array}{l}\text { Minor horizon- } \\
\text { tal principal } \\
\text { stress (MPa) }\end{array}$ & $\begin{array}{l}\text { Vertical stress } \\
(\mathrm{MPa})\end{array}$ \\
\hline 590 & 30.40 & 17.73 & 10.44 \\
1044 & 37.99 & 20.77 & 21.48 \\
\hline
\end{tabular}

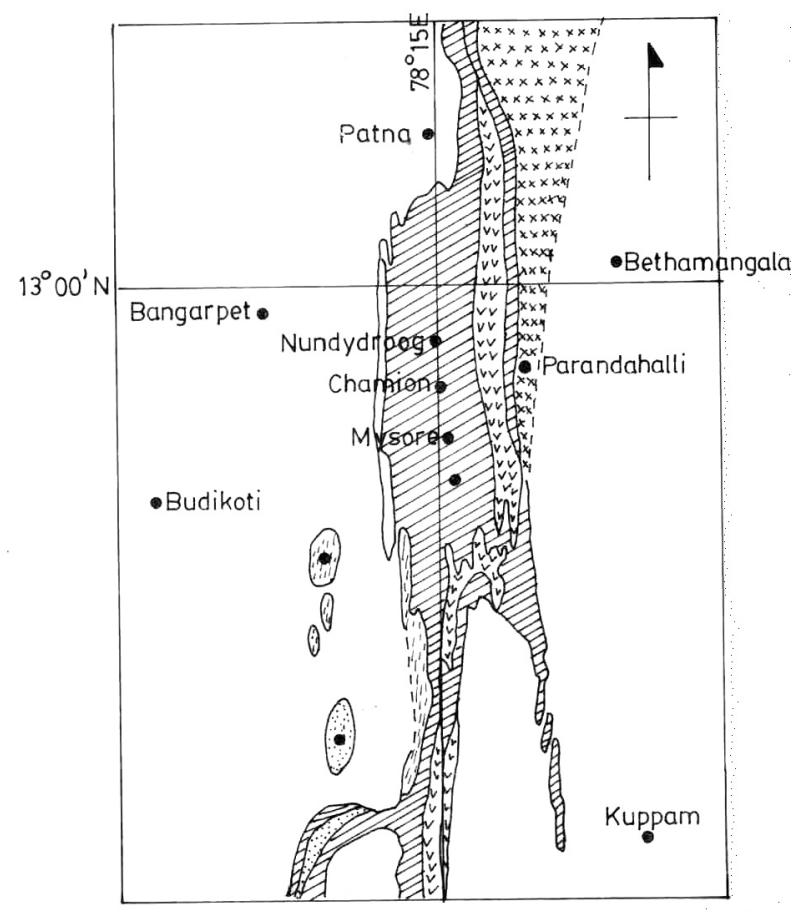

HORNBLEDE SCHIST

GRANITE GNEISS

YY. CHAMPION GNEISS

HORNBLENDE GNEISS

QUARTZITE

GNEISS

\section{FIG. 1 Kolar Gold Field Geological Map}

\section{MINING ACTIVITY}

The Gold mining at KGF dates back to $17^{\text {th }}$ Century wherein the workings extended up to $100 \mathrm{~m}$ depth below the surface. With increased investment, deeper levels were reached with better grades. The deepest working point extended up to $3230 \mathrm{~m}$ depth below surface. Mining at these ultra depths presented unique problems including the problem of

rockbursts and ground control. The first recorded rockburst in one of the KGF mines was in 1898, in a stope below $960 \mathrm{ft}$ level (Taylor, 1955). The rockburst problem has been critical at greater depths, particularly when the ore body to be mined was associated with faults, dykes and pegmatite.

The extraction of lode in Champion reef mine was done mainly by i) bottom stoping with granite support, ii) stope drive with concrete support and iii) flatback stoping with sand fill. The rill system of stoping that was practiced earlier at great depths was subsequently replaced by stope driving.

Two main recent working areas in Champion reef mine were GLEN ORE SHOOT (Southern side) and NORTHERNFOLDS (Northern side) with level interval of $23 \mathrm{~m}$ and $30 \mathrm{~m}$ (Fig.2).

The Glen ore shoot (below 84 level or $2447 \mathrm{~m}$ ) of champion reef mine was mined by the rill system of stoping with a ' $\mathrm{V}$ ' sequence (inverted Christmas tree) with dry granite walling as support. The reef drives and the portions of crosscuts and footwall drives whenever necessary were supported by steel arches, lagged with casuarina poles and filled with waste rocks. This method of stoping was found unsafe with heavy falling of rocks, and later replaced by stope driving.

In the Northern folds (below $73^{\text {rd }}$ level to $113^{\text {th }}$ level), the Z-shaped folded formation of reef (in plan). The three limbs namely, West limb, East limb and Main reef, tend to elongate in depth where the average strike length increases from $60 \mathrm{~m}$ to over $120 \mathrm{~m}$. The Mysore North Fault, which is a major geological feature, lies only a short distance into the hangingwall of the folds. Extraction of the reefs was done on all the limbs by rill system of stoping with granite-pack as the support. Above the 87th level, the three limbs of the northern folds were mined concurrently with main reef in advance of the east and west limb.

\section{SEISMIC DATA OF AREA-ROCKBURSTS}

The seismic data used to investigate the three major arearockbursts of Champion reef mine were collected from the R\&D centre of Bharat Gold Mines Limited, KGF. The data were obtained using a multi-channel seismic network of geophones with established facilities to track and display the seismic activity in the mining area round the clock. The network consisted of 14 velocity transducers deployed on the surface and in underground mines covering a total area of $8 \mathrm{~km}$ length and $3 \mathrm{~km}$ width. The events detected by five or more geophones in a network were used for the location of each rockburst. The error in location of the foci of rockburst was less than $200 \mathrm{ft}(60 \mathrm{~m})$. Three major rockbursts, viz., area-rockburst-I (ARB-I), area-rockburst-II (ARB-II) and area-rockburst-III (ARB-III) of Champion Reef mine, were chosen for the study because they have been associated with different mining and geological conditions. The ARB-I was a shallow one (around $1000 \mathrm{ft}$ or $303 \mathrm{~m}$ depth) and was not much affected by major geological features; whereas ARB-II was a deep seated one (around 10,000 ft or $3030 \mathrm{~m}$ depth) and was affected by major geological features such as folds, fault and pegmatite intrusions.

ARB-I consisted of a series of tremors occurring in abandoned and exhausted regions of Champion Reef mine. During the first hour following the major rokburst of local magnitude 2.48 , about 38 rockburst tremors were detected by five or more geophones in the network. During the next 14 days, as many as 125 rockburst tremors of major to medium intensity in a shallow level covering a strike length of approx. $2500 \mathrm{ft}$ (or $757.6 \mathrm{~m}$ ) were recorded from the region-I (Fig. 2). The foci of the rockbursts were scattered in virgin granite and involved stopes containing pillars and remnant pillars. The ARB-II consisted of a series of tremors of 64 events of minor to medium intensity that occurred after the major rockburst of local magnitude 2.44 over a period of about one week in 
the region-II (Fig.2). The foci of ARB-II were concentrated in the form of clusters along the current stoping regions at deeper levels of the Northern fold area $(11,000 \mathrm{ft}$ or $3333 \mathrm{~m})$. The majority of rockbursts had their foci clustered along the current stoping regions. The ARB-III also consisted of a series of tremors (66 events) of minor to medium intensity followed by a major rockburst of local magnitute 3.09 in the region-III (Fig. 2). These events took place for a period of 15 days in the old workings where mining was abandoned in the early forties. The time gap between ARB-I and ARB-II was around three years, whereas the time gap between ARB-II and ARBIII was around 20 days.

\section{GENERALISED CORRELATION INTEGRAL FUNCTIONS}

A multifractal is considered to be inter-woven with infinitely many sub-fractal sets of different dimensions. The approach involves a whole spectrum of non-integer dimensions of the set under consideration. Generalised dimensions (or multifractal dimensions) contain information about concentration of clustering properties and intermittence of spatial distribution (Geilikman et al. 1990, Hirabayashi et al. 1992) and can be used to distinguish between a homogeneous and heterogeneous fractal set. Following Grassberger (1983), Hentschel and Procaccia (1983) and Hirata and Imoto (1991), the generalised dimension, $D_{q}$ is defined as:

$$
D_{q}=\frac{1}{(q-1)} \lim _{L} \frac{\log \sum_{i \in L} P_{i}^{q}}{\log L}
$$

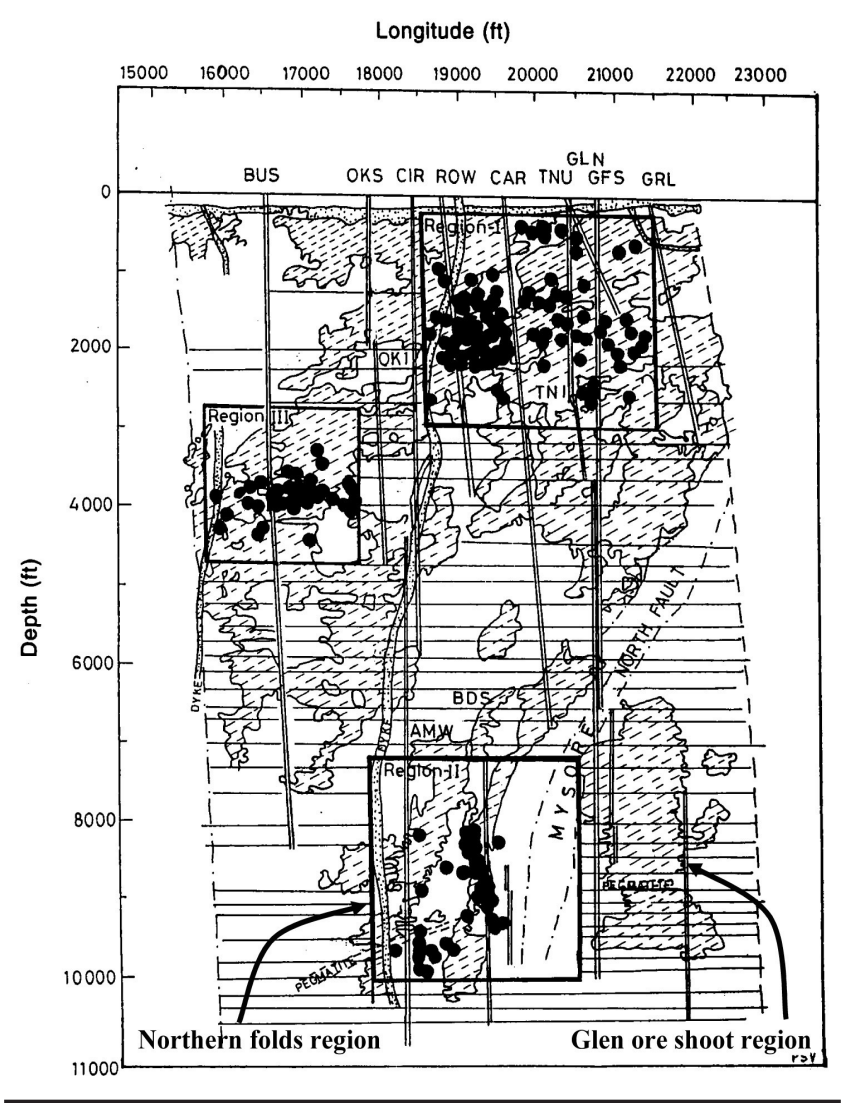

FIG. 2 A longitudinal section showing major geological features, and the hypocenter distribution of area-rockbursts I, II and III of Champion reef mine. Two parallel lines represent shafts. Hatched lines indicate excavated area
Where $P$ is the probability that spatial points fall into a box with size $L$. The parameter $q$ can take a value ranging from $-\infty$ to $\infty$.. Among the various methods to estimate the value of $D_{q}$, the generalised correlation integral method given by Kurths and Herzel (1987) and Hirata and Imoto (1991) was used in this study. A generalised correlation integral function is given by:

$$
C_{q}(r)=\left(\frac{1}{N} \sum_{j=1}^{N}\left(n_{j}(r)\right)^{q-1}\right)^{1 /(q-1)}
$$

Where $N$ is the total number of hypocenters, and $n_{j}(r)$ is a local density function defined as:

$$
\begin{aligned}
& n_{j}(r)=\frac{1}{N-1} \sum_{j \neq k} \Theta\left(r-\left|x_{j}-x_{k}\right|\right), \\
& \Theta(s)=\left\{\frac{1}{0} \frac{\text { if } S \geq 0}{\text { otherwise, }}\right.
\end{aligned}
$$

$\left|x_{j}-x_{k}\right|$ is the distance between the two event pairs $\left(x_{j} x_{k}\right)$.

$$
c_{q}(r) \propto r^{D_{q}}
$$

If the spatial distribution of the hypocenters of arearockburst is fractal, then $C_{f}(r)$ against $r$ must obey the power law in equation (3), and the power exponent denotes its fractal dimension. In the case of a homogeneous fractal, $D_{0}=D_{1}=D_{2}=\ldots=D_{\infty^{\prime}}$ and for heterogeneous fractal, $D_{0} \geq D_{1} \geq D_{2} \geq \ldots \geq D_{\infty} . \quad D_{\infty}$, which is the lower limit of fractal dimension elucidating information about the most intensive clustering in the heterogeneous fractal set. The difference $\left(D_{2}-D_{\infty}\right)$ represents the degree of heterogeneity and $D_{2}$ coincides with the correlation dimension.

\section{MULTIFRACTAL STRUCTURE OF AREA- ROCKBURSTS}

The multifractal structure of the spatial distribution of ARB-I, II and III was analysed using the above generalised correlation integral functions. Generalised dimensions, $D_{q^{\prime}}$ of the spatial distribution of rockbursts were calculated from the slopes of generalised correlation integrals, $C_{q}(r)$ vs distance $r$ on a log-log plot by least square method for positive values of $q$, which ranges from 2 to 10 (Figs 3-5). The correlation integral functions have shown that the spatial distribution of hypocenters of all the three area-rockbursts has a multifractal structure. With the increase of $q$, the change in $D_{q}$ reduces and when $q \geq 10, D_{q}$ changes slightly. Hence, it is reasonable to use $D_{10}$ as an approximation for $D_{\infty}$. The self-similarity of hypocenters of all the three area-rockbursts is apparently valid for the scale range studied (around $200 \mathrm{ft}$ or $60 \mathrm{~m}$ to $1000 \mathrm{ft}$ or $303 \mathrm{~m})$. $C_{q}(r)$ for values of $r$ less than $200 \mathrm{ft}$ was not determined as the accuracy of the hypocenter location of rockbursts was limited to $200 \mathrm{ft}$. The spatial distribution of all the three area-rockbursts indicates that they are heterogeneous with generalised dimension $D_{2}=2.10>D_{3} \ldots>D_{\infty}=1.58$ for ARBI and $D_{2}=1.45>D_{3} \ldots>D_{\infty}=1.08$ for ARB-II and $D_{2}=1.95>D_{3} \ldots$ $>D_{\infty}=1.54$ for ARB-III. The degree of heterogeneity $\left(D_{2}-D_{\infty}\right)$ in the cases of ARB-I, II and III are $0.52,0.37$ and 0.41 respectively.

\section{DISCUSSION}

The spatial pattern of seismicity is controlled by the state of stress distribution and the heterogeneity of the strength distribution in the rockmass. Since the fractal dimension characterises the spatial pattern of seismicity, one can predict some of the fracture characteristics by knowing the fractal dimension. If $D_{q}=3$, the spatial distribution would be uniform and randomly distributed. If $D_{q}=2$, the 
spatial distribution would be planar and if $D_{q}=1$, the spatial distribution would be a linear feature in three-dimensional space. Also a homogeneous stress field will produce a more random spatial distribution of fractures with higher fractal dimension, whereas a heterogeneous stress field will produce a cluster of fractures with lower fractal dimension (Lei et al. 1993).

In our multifractal analysis, the spatial distribution of arearockbursts indicates that they are heterogeneous fractals with generalised dimension $D_{2}=2.10>D_{3} \ldots>D_{\infty}=1.58$ for ARB-I, and $D_{2}=1.45>D_{3} \ldots>D_{\infty}=1.08$ for ARB-II, and $D_{2}=1.95>D_{3} \ldots>D_{\infty}$ $=1.54$ for ARB-III. The heterogeneous fractal structure might describe the distribution of the damage regions or destressed regions. The differences in fractal dimension in all the three area-rockbursts are due to difference in the spatial pattern of seismicity. The differences in the spatial pattern of seismicity indicate that they are bounded by different heterogeneous stress fields which are associated with different mining and geological conditions. The ARB-I has shown a multifractal structure with degree of heterogeneity $\left(D_{2}-D_{\infty}\right)$ equal to 0.52 . The large difference between $D_{2}$ and $D_{\infty}^{\infty}$ shows that the distribution of seismic cluster is highly heterogeneous compared to the other area-rockbursts, even though the region is not affected by any major geological features. During the developing stoping operations carried out several decades ago, rockbursts had frequently occurred resulting in several pillars being left behind, and the region was abandoned. Due to time dependent strain effects over a period of time, these pillars appear to have failed in that region resulting in regional redistribution of stress, causing heterogeneous spatial distribution of hypocenters with many sub-fractal sets of different dimensions.

The ARB-II had taken place at a deeper level in the region of high stress. The multifractal dimensions of ARB-II are relatively low compared to other area-rockbursts studied. This is reasoned from the fact that the region where the ARB-II took place has been known to contain high stress due to the presence of the Mysore North Fault, pegmatite intrusion and folded nature of the lode. The working stopes, which were close to these geological features at the deeper levels, have further enhanced the stress in that region in the form of an area-rockburst. The multifractal analysis of this region indicates heterogeneous fractal structure with a degree of heterogeneity $\left(D_{2}-D_{\infty}\right)$ of 0.37 . Although we find heterogeneity in the rockmass due to the presence of major geological features, the heterogeneity in the spatial distribution of the hypocenters of area-rockbursts is relatively less compared to the ARB-I. It appears that the presence of Mysore North Fault must have enhanced the stress in the region resulting in a dense cluster in the spatial distribution and lower multifractal dimensions.

The lower $D$ of the region characterises the non-uniform stress field (Shivakumar et al. 1996). Karekal et.al (1996) observed a heterogenous fractal structure and lower $D_{q}$ $\left(D_{2}=1.49>D_{3} \ldots>D_{\infty}=1.00\right)$ during single stage triaxial creep test on amphibolite rock containing pre-existing macroscopic flaws. Lei et al. (1993) have observed relatively higher $D_{q}$ $\left(D_{2}=2.75>D_{3} \ldots>D_{\infty}=2.4\right)$ during triaxial compressional test on Oshima granite containing no macroscopic pre-existing flaws. These experimental observations reveal that heterogeneity can lead to a more non-uniform stress distribution and lower multifractal dimensions. In the case of ARB-II, the intense clustering characterised by lower $D$ in the region-II must have reduced the stiffness of the region causing re-distribution of stress in the surroundings.

The ARB-III occurred immediately following the ARBII. The region where the ARB-III took place contains a few old workings abandoned in the early forties. But there was neither any sign of rock movement nor any seismic activity

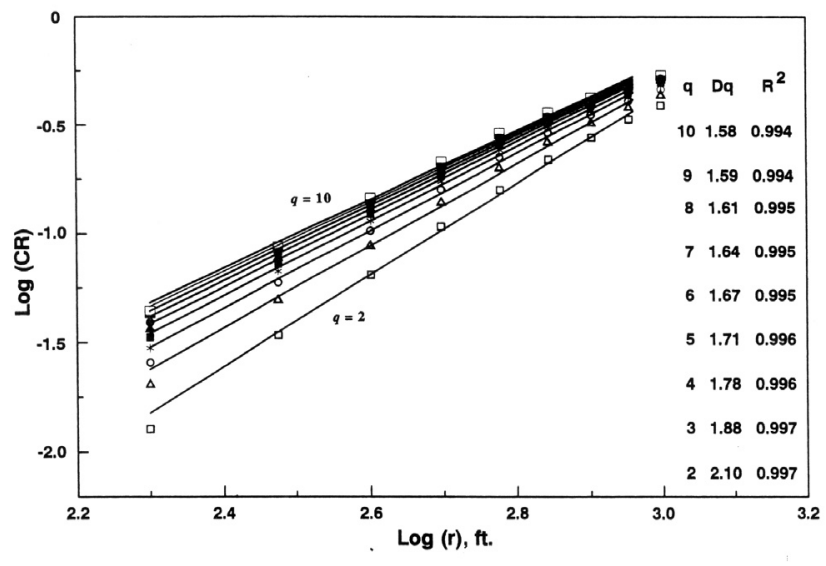

FIG. 3 Generalised correlation integral functions of hypocenter distribution of area-rockburst-I. The fractal dimensions for $q=2-10$ are specified on the right side along with its correlation coefficient $\left(R^{2}\right)$

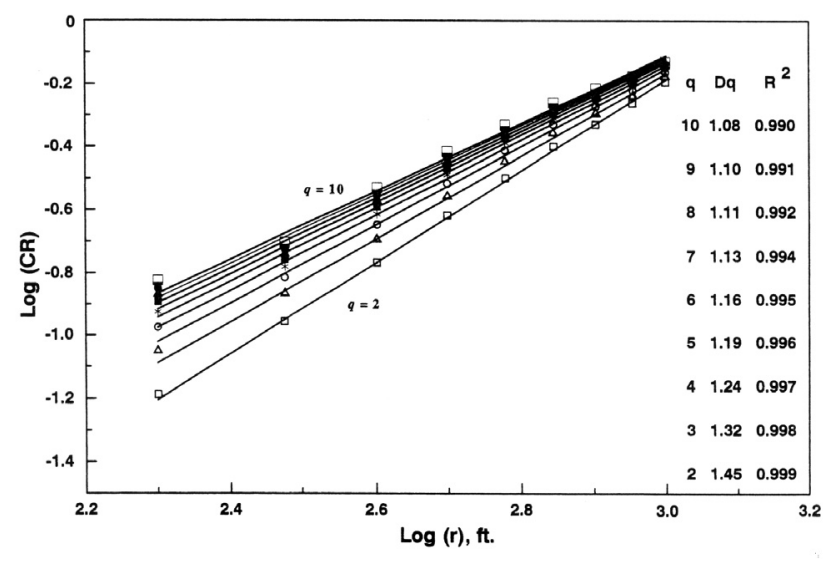

FIG. 4 Generalised correlation integral functions of hypocenter distribution of area-rockburst-II. The fractal dimensions for $q=2-10$ are specified on the right side along with its correlation coefficient $\left(R^{2}\right)$

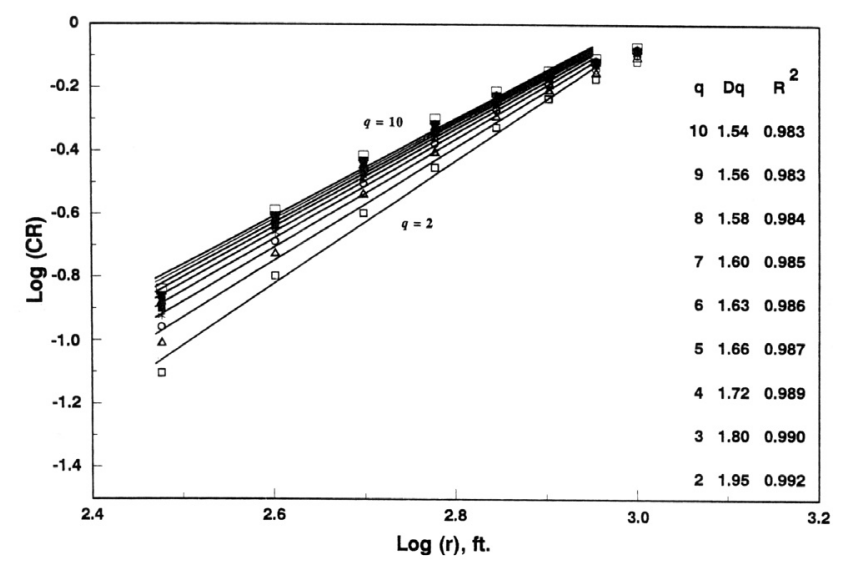

FIG. 5 Generalised correlation integral functions of hypocenter distribution of area-rockburst-III. The fractal dimensions for $q=2-10$ are specified on the right side along with its correlation coefficient $\left(R^{2}\right)$ 
for a few years prior to the occurrence of ARB-III in that region. The destressing of the region-I and region-II must have contributed to the enhancement of the stress in the region-III to a critical state, causing the ARB-III to occur. Multifractal analysis of the hypocenters of ARB-III had also shown a multifractal structure having the degree of heterogeneity of the spatial distribution of burst events equal to 0.41 . The heterogeneous fractal structure is probably due to the clustering of events around old excavations, which have made it heterogeneous, and a high stress region. The multifractal dimension characterised by $D_{q}$ are higher than those of the ARB-II, indicating that the stress distribution is relatively less heterogeneous. The cluster of events of ARB-II appears to have migrated from region-II to region-III, but not to the other side of the Mysore North Fault where the mining activities were going on in Glen oreshoot (Fig.2). This could be attributed to the fact that the Mysore North Fault acted as a free surface and thus did not transmit stresses across.

\section{SUMMARY}

The spatial pattern of seismicity is controlled by the state of stress distribution and the heterogeneity of the strength distribution in the rockmass. The spatial distributions of hypocenters of the area-rockbursts have shown to be heterogenous fractal. The fractal characteristics of arearockbursts were distinctly different in each region, and were mainly governed by the state of stress distribution that resulted from mining and geological conditions (regional heterogeneity). Presence of fault resulting in a higher stress concentration in its vincinity and thereby restricting the spread of fractal dimensions (generally confined to lower values). The multi-fractal technique can also be used to characterise the complex spatial distribution of microseismic events that are associated with rockbursts, in a similar manner as described in this paper. The variations in the correlation dimensions and the degree of heterogeneity $\left(D_{2}-D_{\infty}\right)$ values with time could then be used as premonitors for predicting impending rockbursts.

\section{REFERENCES}

Cook, N.G.W. (1976) Seismicity associated with mining. Engng Geol, pp. 99-122.

Coughlin, J. and Kranz, R. (1991). New approaches to studying rockburst associated seismicity in mines. In: Proc. 32nd U.S. Symp. Rock Mech. pp. 491-500 Balkema, Rotterdam.

Geilikman, M.B., Golubeva, T.V. and Pisarenko, V.F. (1990) Multifractal patterns of seismicity. Earth Planetary, Sci. Lett. 99, pp. 127-132.

Gowd, T.N., Rao, M.V.M.S., Krishnamurthy, R,. Rummel, F. and Alheid, R. (1981) A Indo-German collaboration project report on Insitu measurements by hydro-fracturing in the underground mines of Kolar Gold Fields, India.

Grassberger, P. (1983) Generalized dimension of strange attractors. Phys. Let. 97A, pp. 227-230.

Hentschel, H.G.E. and Procaccia, I. (1983) The infinite number of generalized dimensions of fractals and strange attractors. Physica 8D, pp. 435-444

Hirabayashi, T., Ito, K. and Yoshi, T. (1992) Multifractal analysis of earthquakes. Pageoph 138, pp. 591-610.

Hirata, T., and Imoto, M. (1991) Multifractal analysis of spatial distribution of micro-earthquakes in the Kanto region. Geophys. J. Int. 107, pp. 155-162.

Hirata, T., Satoh, T. and Ito, K. (1987) Fractal structure of spatial distribution of microfracturing in rock. Geophys. J. R. Astron. Soc. 90, pp. 369-374.

Karekal, S., Satoh, T., Nishizawa, O., Kusunose, K. and Rao, M.V.M.S. (1994) Micromechanics of fracturing of jointed amphibolite as inferred from spatio-temporal distribution of AE hypocentres and surface strain map. National Institute of Rock Mechanics Interim Report \#2, IJPC/93-95.

Krishnamurthy, R. (1977) Investigations into the stability and design of workings in deep mines. In: (2nd edn.) J. Mines, Metals \& Fuel, Spl. Vol. Proc. BPE Seminar-I, pp. 80-87.

Krishnamurthy, R. (1986) Report on the Area Rockbursts in the Champion Reef Mine. Bharat Gold Mines Limited Interim Report, file No: 20-C-832.
Krishnamurthy, R. and Shringarputale, S.B. (1990) Rockburst hazards in Kolar Gold Fields. In: Proc. 2nd Symp. Rockburst \& Seismicity in Mines, pp. 410-411 Balkema, Rotterdam.

Kurths, J. and Herzel, H. (1987) An attractor in a solar time series. Physica 25D, pp. $165-172$.

Lei, X., Nishizawa, O. and Kusunose, K. (1993) Band-limited heterogeneous fractal structure of earthquakes and acoustic-emission events. Geophys. J. Int. 115, pp. 79-84.

Mandelbrot, B.B. (1982) The Fractal Geometry of Nature. Freeman, San Francisco.

Miller, E. (1967) Notes on rock mechanics research in the Kolar Fields. KGF Min. Met. Soc. Bull. 23, pp. 23-61.

Mogi, K. (1962) Magnitude-frequency relation for elastic shocks accompanying fractures of various materials and some related problems in earthquake. Bull. Earthquake Res. Inst. Univ. Tokyo 40, pp. 831-853.

Scholz, C.H. (1968) Microfractures, aftershocks and seismicity. Bull. Seismol. Soc. Am. 58, pp. 1117-1130.

Shivakumar, K., Rao, M.V.M.S., Kusunose, K., Satoh, T. and Nishizawa, O. (1996) Microfracturing process and failure zone development in jointed amphibolite rock as observed from acoustic emission source locations and surface strain mapping. Proc. 14th World Conf. On NDT, New Delhi, India, pp. 2459-2462.

John Taylor and Sons (India) Ltd (1955) Report of the special committee on the occurrence of rockbursts in the mines of the Kolar Gold Fields, Mysore State, South India.

Xie, H. and Pariseau, W.G. (1992). Fractal character and mechanism of rockbursts. In: Proc. 33rd U.S. Symp. Rock Mech., pp. 745-754 Balkema, Rotterdam. 